Arteterapia. Papeles de arteterapia y educación para inclusión social ISSN-e 1988-8309

http://dx.doi.org/10.5209/ARTE.59743

\title{
Proyecto ARPA:Arteterapia con personas amputadas. Resultados, reflexiones ético-metodológicas y conclusiones del estudio ${ }^{1}$ Kathya Adsuar Pascual2 ${ }^{2}$, Javier Serrano Carballo ${ }^{3}$
}

Recibido: 30 de marzo de 2018 / Aceptado: 18 de julio de 2018

Resumen. El proyecto Arpa es un estudio piloto de investigación cuantitativa llevado a cabo en la Unidad de Cirugía Vascular del Hospital del Mar y centro Fórum de Barcelona durante los años 2016 y 2017. En el presente artículo se expone el desarrollo, resultados y limitaciones del mismo, así como las dificultades encontradas y las cuestiones ético-metodológicas derivadas.

El estudio ha ofrecido sesiones de Arteterapia a 9 personas intervenidas quirúrgicamente por una amputación. Los resultados obtenidos muestran mejoras en síntomas de depresión y autoimagen y datos contradictorios en autoestima y respuestas psicológicas de duelo. El cuestionario diseñado por el equipo indica una satisfacción alta aunque inespecífica de la atención recibida.

Se concluye la presencia de efectos positivos, si bien son necesarios estudios con muestras de mayor tamaño y rigurosidad en su metodología.

Palabras clave: arteterapia; amputación; duelo; cirugía vascular.

\section{[en]ARPAProject: Art therapy with amputees. Results, ethical-methodological reflections and conclusions of the study}

\begin{abstract}
The Arpa project is a pilot study of quantitative research carried out in the Vascular Surgery Unit of the Hospital del Mar and the Fórum de Barcelona center during the years 2016 and 2017. In this article the development, results and limitations of it are exposed, as well as the difficulties encountered and the ethical-methodological issues derived.

The study has offered Art Therapy sessions to 9 people surgically treated for an amputation. The results obtained show improvements in symptoms of depression and self-image and contradictory data on selfesteem and psychological responses to grief. The questionnaire designed by the team indicates a high, although non-specific satisfaction with the care received.

The presence of positive effects is concluded, although studies with larger and more rigorous samples in their methodology are necessary.
\end{abstract}

Keywords: art therapy; amputation; duel; vascular surgery.

Sumario: Introducción. Justificación. Finalidad y objetivos. Diseño. Resultados. Discusión.. Limitaciones del estudio. Reflexiones ético-metodológicas. Apuntes cualitativos. Conclusiones. Referencias.

\footnotetext{
Proyecto realizado con el apoyo de la Fundación de Amigos del Hospital del Mar.

2 Diplomada en Terapia Ocupacional, Máster en Arteterapia por la Universidad de Girona y miembro profesional de Ate (Asociación Profesional Española de Arteterapeutas)

k.adsuar@gmail.com

3 Diplomado en enfermería en la Escuela Universitaria de Enfermería del Mar. Posgrado de Enfermería Comunitaria, de la Escuela Universitaria de Enfermería de Vall d'Hebron de Barcelona. Enfermero de la Unidad de Cirugía Vascular del Hospital del Mar, donde realiza investigación en el proyecto ARPA. vdraco_bcn83@hotmail.com
} 
Cómo citar: Adsuar Pascual, K., Serrano Carballo, J. (2018). Proyecto ARPA: Arteterapia con personas amputadas. Resultados, reflexiones ético-metodológicas y conclusiones del estudio, en Arteterapia. Papeles de arteterapia y educación para inclusión social 13, 2018, 33-52.

\section{Introducción}

La Unidad de Cirugía Vascular del Hospital del Mar atiende a personas mayoritariamente con patología arterial o complicaciones de la diabetes, que en muchos casos acaban sufriendo amputaciones quirúrgicas. Además de los cambios físicos, funcionales y sociales que esta situación provoca, este tipo de pacientes suele presentar alteraciones psicológicas que habitualmente requieren de un abordaje específico.

Además de los tratamientos tradicionales, existen nuevas terapias que comienzan a generar interés para el abordaje de esta problemática. Entre ellas se encuentra el arteterapia, disciplina que a lo largo de los últimos años ha ido aumentando su presencia en muy variados campos de intervención.

En este contexto diseñamos el proyecto ARPA (Arteterapia con personas amputadas), estudio piloto de investigación cuantitativa que ofrece sesiones de arteterapia en dispositivo individual a personas intervenidas de una amputación. A finales del año 2015 el estudio se somete a la evaluación del Comité Ético de Investigación Clínica del Parc de Salut Mar de Barcelona, obteniendo su aprobación. El proyecto cuenta además con el apoyo de la Fundación de Amigos del Hospital del Mar.

\section{Justificación}

La amputación ${ }^{4}$ se define como la extirpación quirúrgica de una parte del cuerpo, de una extremidad o parte de ella, que se efectúa para tratar infecciones recurrentes o gangrena en las enfermedades vasculares periféricas, para extirpar tumores malignos $\mathrm{y}$ en los traumatismos graves.

España es el segundo país del mundo con más amputaciones a causa de la diabetes, quedando sólo por detrás de los Estados Unidos ${ }^{5}$ y se estima que el $80 \%$ del total de amputaciones practicadas anualmente, son debidas a una enfermedad arterial oclusiva.

La realización de una amputación supone un cambio vital altamente significativo. La variación en la capacidad funcional suele derivar en una influencia negativa en el rol social, familiar y personal (Espinoza \& García, 2014; Tonon da Luz et al., 20112) La magnitud de este impacto está condicionada por la puesta en marcha de medidas de rehabilitación, que tengan en cuenta las posibilidades de independencia y reinserción social (Espinoza \& García, 2014). Además, dos aspectos especialmente relevantes en la calidad de vida posterior son la presencia de dolor y el impacto psicológico.

El dolor es una de las secuelas más frecuentes a largo plazo, especialmente el denominado dolor del miembro fantasma, con una incidencia entre el $30 \%$ y el $50 \%$ de

4 Diccionario Mosby de medicina, enfermería y ciencias de la salud. Volumen 1, sexta edición, pag 79. Editorial Elsevier 2003.

5 https://www.redaccionmedica.com/secciones/medicina-interna/espana-segundo-pais-del-mundo-con-masamputaciones-inferiores-por-diabetes-1696 
los casos (Jiménez, Ramírez, Rueda, \& Díaz, 2017). Por otro lado, el fuerte impacto psicológico se traduce en un alta frecuencia de ansiedad y depresión, que según los estudios varía entre el $19-25 \%$ en el caso de la ansiedad y $20-35 \%$ en el caso de la depresión (Atherton, 2002; Ghous, Gul, Siddiqi, Pervaiz, \& Bano, 2015). Otro aspecto relevante de la amputación es el importante cambio en la imagen corporal, que en muchos casos deriva en sentimientos de vergüenza y alteraciones de la autoestima relacionados con la no adaptación a los estándares estéticos (Tizón, 2004).

Así, el cambio en la independencia funcional, la presencia de dolor y el impacto psicológico, social y laboral, genera sufrimiento en cotas que no están necesariamente relacionadas con el nivel ni la magnitud de la amputación (Agea, Luis, Costa, \& Díaz, 2013). En la amputación se produce por tanto una pérdida multidimensional, resultando un evento que según la definición de diversos autores, desencadena un proceso de duelo (Porta \& Retes, 2013).

En un estudio de la Universidad Católica de Murcia que indaga sobre la calidad de vida de personas amputadas, Jiménez et al., (2017) hallaron que el 53.7\% de su muestra (28 personas) tiene una mala calidad de vida y el $82,14 \%$ tiene problemas de salud mental. Sin embargo, sólo el $28.6 \%$ acude a terapia psicológica a pesar de que el $67.9 \%$ muestran interés en recibir información sobre terapias complementarias. Concluyen que el tratamiento psicológico es fundamental y que son necesarias más investigaciones al respecto.

En otro estudio llevado a cabo en Guatemala (Velásquez, 2015) se entrevistó a 20 personas amputadas con diabetes. El estudio indaga sobre las emociones que se producen al recibir la noticia del evento y durante la rehabilitación posterior. En sus hallazgos muestra que la tristeza y la culpa son las más frecuentes manifestaciones. Durante la rehabilitación las personas refieren también importantes niveles de cansancio físico y emocional. En sus conclusiones destaca la importancia de ofrecer apoyo psicológico en todas las etapas del proceso de duelo por la pérdida de salud y no sólo en la fase de hospitalización.

En la Universidad Autónoma del estado de México, Reza, Rodríguez, \& Zarza, (2017) realizaron un estudio descriptivo para conocer el proceso de duelo que afrontan las personas amputadas. Realizaron entrevistas semi-estructuradas y administraron test proyectivos en una muestra de 4 participantes cuya etiología era la diabetes en la mitad de los casos. En sus hallazgos destacan la tristeza y la ira como principales reacciones a la noticia y mayor dificultad para expresar emociones de vergüenza y enfado en el caso de las personas con enfermedad previa. En sus conclusiones destacan también una disminución en la aceptación de su cuerpo no sólo por sus capacidades funcionales, sino relacionado también con el aspecto y atractivo físico.

En el tratamiento integral de este tipo de pacientes, además del abordaje farmacológico podemos distinguir toda una serie de terapias complementarias como la acupuntura, la psicoterapia o la terapia conductual. Los beneficios de las mismas han sido objeto de investigaciones científicas con buenos resultados (Malavera, Carrillo, Gomezese, García, \& Silva, 2014). Sin embargo, son pocas las pacientes ${ }^{6}$ que recurren a estas alternativas, aunque reportan interés en tener más información sobre ellas (Jiménez et al., 2017).

6 Utilizaremos en el presente trabajo el femenino como genérico, haciendo referencia al sujeto "persona», debido a la falta de una normativa que permita utilizar el genérico sin establecer una marca que conlleve a discriminaciones por razón de género. 
Al respecto del uso del arteterapia, no encontramos estudios centrados específicamente en personas amputadas. En un estudio de caso publicado por Orgilles, (2015) se presenta a un paciente que dentro de un entramado de dificultades amplio presenta, además, una amputación debida a un accidente. Sin embargo, esta característica no supone el foco del estudio.

Sí hallamos estudios que sugieren la eficacia del Arteterapia en muy variados campos de aplicación (Case y Dalley, 1992); (Pounsett, et al, 2006); (Luzzato, Sereno y Capps, 2003). En el caso que nos ocupa, resultan especialmente interesantes los llevados a cabo por Collette, $(2011,2016)$ en pacientes en proceso de final de vida. El trabajo llevado a cabo por esta autora tiene similitudes con nuestro estudio; se produce en el ámbito hospitalario, en dispositivo individual y en él está muy presente el proceso de duelo por la pérdida de salud.

\section{Finalidad y objetivos del estudio}

La finalidad del proyecto ARPA es aportar conocimiento sobre el uso del arteterapia en pacientes intervenidos de una amputación, lo cual puede contribuir a la generación de nuevas líneas de intervención para mejorar la calidad de vida de estas personas.

Para ello, la investigación trató de responder a la siguiente pregunta:

¿Puede el Arteterapia ser efectiva para:

- Favorecer el proceso de duelo no patológico?

- Disminuir la presencia de síntomas depresivos?

- Fortalecer la autoestima?

- Mejorar la elaboración de una autoimagen completa?

\section{Diseño}

Para la consecución de nuestros objetivos, diseñamos un estudio con asignación no aleatoria, prospectivo, pre-post intervención. No se dispone de muestra de control al no poder alcanzar una muestra que lo permitiese. La duración del estudio se extiende a lo largo de 2 años y en su diseño podemos diferenciar varias fases:

\section{Fase I: Selección de la muestra.}

La selección de participantes se lleva a cabo en la unidad de cirugía vascular del Hospital del Mar de Barcelona con los siguientes criterios:

Criterios de inclusión:

- Personas adultas intervenidas quirúrgicamente de una amputación independientemente de su magnitud.

- Previsión de hospitalización de al menos un mes.

- Recomendación de participación por parte del equipo de enfermería.

Criterios de exclusión:

- Barrera idiomática o grave afectación del estado cognitivo que no permita la valoración pre-post test. 
- Estado de salud inestable que no permita la intervención arteterapéutica (por ejemplo cotas altas de dolor).

\section{Fase II: Inclusión en el estudio.}

Una vez revisados los criterios de inclusión, se pone en marcha la incorporación al estudio. Este se desarrolla de la siguiente forma: una profesional del equipo de enfermería comunica a la persona que recibirá una visita para hacerle una propuesta terapéutica, posteriormente la arteterapeuta realiza la entrevista en la que se presenta como tal, explica en qué consiste el proyecto y qué supone participar en él. En este encuentro se especifican:

1. Que la intervención ofrece sesiones de Arteterapia en dispositivo individual dos veces por semana con una hora de duración.

2. Características básicas del trabajo: no es necesario poseer conocimientos artísticos previos, confidencialidad del proceso y estructura de las sesiones.

3. Que se le facilita un documento informativo así como dos documentos de consentimiento informado: el primero relativo a la participación en el estudio, el segundo referido a la toma de fotografías.

4. Que previo al comienzo de las sesiones se administrará una batería de valoración, cuya duración es aproximadamente de media hora.

5. Que el estudio puede abandonarse en cualquier momento que se decida sin necesidad de dar explicaciones.

Tras recibir esta información, se ofrece la resolución de dudas que puedan surgir al respecto. Si la persona acepta participar en el estudio, se pacta una fecha para la valoración pre-test y firma de documentos, así como para el comienzo de las sesiones.

\section{Fase III: Evaluación pre-test.}

Una vez aceptada de forma verbal la participación en el estudio, una profesional del equipo de enfermería facilita los documentos asociados, recoge los datos sociodemográficos y clínicos y administra la batería de valoración. El tiempo que toma este procedimiento es aproximadamente de 30-40 minutos.

Los documentos asociados al estudio son los siguientes:

- Carta informativa: Se recogen los objetivos, ubicación y duración del estudio, así como el carácter voluntario de la participación y el amparo de la ley 15/99 de protección de datos de carácter personal. Asimismo, se explica qué es arteterapia, la duración y periodicidad de las sesiones y la confidencialidad sobre el contenido de estas. Se recoge también que la intervención estará supervisada por otra profesional del ámbito como así lo exige el código ético de la Asociación Profesional Española de Arteterapeutas (Ate) ${ }^{7}$. 
- Consentimiento informado: Que el paciente firma para registrar su conformidad con la participación en el estudio tras haber recibido la carta informativa y haber resuelto las posibles dudas al respecto de la misma.

- Consentimiento informado para la realización de fotografías y su posterior utilización: Que el paciente firma para registrar su conformidad con la toma de fotografías de las producciones artísticas realizadas durante las sesiones de Arteterapia. El fin de las mismas se restringe al uso en ámbitos académicos y divulgativos.

Los datos sociodemográficos y clínicos recogidos son los siguientes:

- Edad.

- Género.

- Diagnóstico médico.

- Motivo de ingreso.

La batería de valoración está formada por las siguientes escalas:

- Escala de Respuestas Psicológicas de Duelo ante la Pérdida de la Salud (RPD-PDS-38) (Miaja Ávila \& Moral de la Rubia, 2014). Conformada por 38 ítems tipo Likert ${ }^{8}$, en los que se engloban reacciones, acciones y sentimientos relacionados con el proceso del duelo y valorados por 5 factores numéricos (1 nunca, 2 pocas veces, 3 a veces, 4 casi siempre y 5 siempre).

- Escala de Beck (Sanz, Perdigón, \& Vázquez, 2003) para detectar la presencia de sintomatología depresiva. Consta de 21 ítems que según el resultado final, determinan el grado de ansiedad que presenta la paciente (baja, moderada o severa).

- Escala de Rosemberg (Góngora \& Casullo, 2009) para valorar la autoestima. Explora los sentimientos de valía personal a través de 10 ítems evaluados mediante una escala Likert (Muy de acuerdo, De acuerdo, En desacuerdo, Muy en desacuerdo). La escala permite detectar desde una autoestima elevada a una autoestima baja.

- Escala de imagen corporal BIS (Juárez \& Landero, 2014) para valorar la percepción de la autoimagen: cómo se siente la paciente en relación a su apariencia, los cambios que ha experimentado en relación a la enfermedad y los tratamientos que le han realizado o le tienen que realizar. Consta de diez ítems evaluados mediante una escala Likert (nada, un poco, bastante y mucho).

- Cuestionario semi-estructurado ${ }^{9}$ diseñado por el equipo con el fin de valorar aspectos más específicos relacionados con la intervención arteterapéutica.

\section{Fase IV: Intervención.}

La intervención ofrecida consiste en sesiones de Arteterapia en dispositivo individual con una duración de una hora y una frecuencia de dos encuentros por semana. En el diseño del proyecto se plantea la realización de sesiones durante dos meses.

Los ítems tipo lickert especifican el nivel de acuerdo o desacuerdo con una declaración El cuestionario puede consultarse en el apartado de resultados. 
En referencia al encuadre terapéutico, se trata de un dispositivo individual. Las sesiones se realizan en una sala del centro facilitada para ello o en la habitación del paciente, lo cual depende de la necesidad o no de permanecer en la cama. Esto supone que en ocasiones la sesión se lleva a cabo con la presencia en la misma habitación de la compañera de la misma. En cada caso, se coordina con la unidad de enfermería para no interrumpir las curas y otros cuidados prioritarios que requieran las participantes.

El material de que disponemos es el siguiente: témperas, rotuladores, bolígrafo y lápiz, plastilina, revistas, y pegamento. Como posibles soportes disponemos de folios y cartulinas de color blanco.

La estructura de las sesiones es la siguiente:

- Acogida: A través de la palabra y de forma no directiva la persona tiene la oportunidad de expresar cómo se siente en ese momento y compartir lo que desee sobre sus vivencias.

- Creación: Tras un tiempo variable según el estado de la persona, se realiza la propuesta artística, cuyo nivel de directividad está en consonancia con lo expresado en la primera parte. En la medida en que la persona lo permite, en ella se pretenden abordar principalmente los temas relacionados con los objetivos del estudio: sintomatología depresiva, autoestima, autoimagen y aquellas experiencias relacionadas con el proceso de duelo.

- Puesta en común y cierre: Se invita a la persona a poner palabras a la experiencia con el material y las emociones que han surgido de la misma, así como las posibles reflexiones que puedan derivarse de ellas. Durante este tiempo la producción realizada está presente y es posible realizar alguna modificación si se desea o ello aporta más sentido a las reflexiones realizadas. Al cierre de la sesión se pide el consentimiento para registrar la producción en una fotografía $^{10} \mathrm{y}$ se recoge tanto el material como las producciones realizadas.

Durante el tiempo de cada sesión, todas las producciones que la persona va realizando se encuentran disponibles para esta, que puede volver a ellas en el momento que lo desee. Fuera de las sesiones, mientras dura el proceso la arteterapeuta custodia tanto las producciones como el material utilizado.

En el cierre de cada proceso se invita a la persona a visualizar las producciones realizadas y a tomar decisiones respecto al destino de estas. En este momento la arteterapeuta facilita el recuerdo de los contenidos abordados y se realiza una reflexión final sobre el trabajo realizado.

\section{Fase V: Evaluación post-test.}

Una vez realizadas las sesiones de arteterapia, una profesional del equipo de enfermería vuelve a pasar las mismas escalas que en la evaluación pre-test. En el cuestionario diseñado por el equipo introdujimos ligeras variaciones con el fin de dar cohe-

10 Este consentimiento se pide expresamente, aunque ya se dispone del documento firmado para ello, pues nos parece un posicionamiento ético más acorde con el respeto a la persona y su derecho a cambiar de opinión en momentos determinados o condiciones concretas. 
rencia a la comparación de respuestas previas y posteriores a la terapia. Por ejemplo, en el cuestionario previo se pregunta ¿cree que la actividad artística le ayudaría a la expresión emocional? y en el cuestionario posterior ¿la actividad artística le ha ayudado a la expresión emocional?

\section{Resultados}

A continuación, presentamos los datos recogidos en el estudio organizados en tres apartados: participación y perfil de las pacientes; resultados de valoración pre-test y comparativa pre-post test de las pacientes que completaron la terapia.

\subsection{Participación y perfil de las pacientes}

Tabla 1. Personas atendidas

\begin{tabular}{|l|r|r|}
\hline ENTREVISTAS PREVIAS: & 12 & $100 \%$ \\
\hline Aceptación de la terapia & 10 & $83.3 \%$ \\
\hline PERSONAS ATENDIDAS: & 9 & $75 \%$ \\
\hline Pérdida por alta & 2 & $22.2 \%$ \\
\hline Abandono & 2 & $22.2 \%$ \\
\hline Empeoramiento salud & 1 & $11.1 \%$ \\
\hline Terapia completa & 4 & $44.4 \%$ \\
\hline
\end{tabular}

Como puede observarse en la tabla 1 la muestra total fue de 12 potenciales participantes, de las cuales $10(83.3 \%)$ aceptaron la inclusión en el estudio. Sin embargo, sólo se llegaron a realizar sesiones con 9 de ellas (una de las participantes fue dada de alta antes de comenzar las sesiones). Del total de personas atendidas 2 de ellas decidieron abandonar el estudio y otras 3 no pudieron realizar la terapia completa por alta del centro o empeoramiento en su estado de salud. Las 4 personas restantes completaron la terapia. En total se realizaron 60 sesiones de Arteterapia. 
Tabla 2. Variables sociodemográficas $(n=10)$

\begin{tabular}{|l|c|c|}
\hline Edad media & 62.9 & \\
\hline Género & 7 & $70 \%$ \\
\hline Varones & 3 & $30 \%$ \\
\hline Mujeres & 5 & $50 \%$ \\
\hline Diagnóstico médico & 3 & $30 \%$ \\
\hline Isquemia crónica & 2 & $20 \%$ \\
\hline Pie diabético & \multicolumn{2}{|l|}{} \\
\hline Shock séptico & 5 & $50 \%$ \\
\hline Motivo de ingreso & 5 & $50 \%$ \\
\hline Amputación mayor &
\end{tabular}

Los datos sociodemográficos recogen una edad media de casi 63 años y mayor presencia de varones. El principal diagnóstico médico fue la isquemia crónica (derivada de enfermedades vasculares), seguido de la diabetes; es decir, dentro de procesos de enfermedad previos. En dos de los casos la amputación se produjo de forma más súbita e inesperada a causa de un shock séptico de origen infeccioso. La magnitud de las amputaciones se reparte en la misma medida entre mayores (aquellas que se producen por encima del tobillo) y menores (producidas por debajo del mismo). En todos los casos la intervención quirúrgica se había producido en un tiempo muy cercano a la inclusión en el estudio (menos de un mes).

\subsection{Resultados de valoración pre-test}

Tabla 3. Resultados escalas de valoración pre-test $(\mathrm{n}=10)$

\begin{tabular}{|c|c|c|}
\hline \multicolumn{3}{|c|}{ BECK (depresión) } \\
\hline & $\mathrm{N}^{\mathrm{o}}$ & $\%$ \\
\hline Sintomatología mínima & 6 & 60 \\
\hline Sintomatología leve & 1 & 10 \\
\hline Sintomatología moderada & 0 & 0 \\
\hline Sintomatología grave & 3 & 30 \\
\hline \multicolumn{3}{|c|}{ ROSENBERG (autoestima) } \\
\hline & $\mathrm{N}^{\mathrm{o}}$ & $\%$ \\
\hline Autoestima baja & 2 & 20 \\
\hline Autoestima media & 3 & 30 \\
\hline Autoestima alta & 5 & 50 \\
\hline \multicolumn{3}{|c|}{ BIS (percepción imagen corporal) } \\
\hline & $\mathrm{N}^{\mathrm{o}}$ & $\%$ \\
\hline Completa & 8 & 80 \\
\hline Incompleta & 2 & 20 \\
\hline
\end{tabular}


Como mostramos en la tabla 3 , los resultados obtenidos en las escalas de depresión y autoestima, concuerdan con los hallazgos de otros autores (Atherton, 2002; Ghous, et al., 2015; Tizón, 2004), con valores en el 40\% en el caso de sintomatología depresiva y $50 \%$ en el caso de autoestima media o baja. La percepción de la imagen corporal es incompleta en el $20 \%$ de los casos.

Tabla 4. Resultados escala (RPD-PDS-38) de valoración pre-test ( $\mathrm{n}=10)$

Escala de Respuestas Psicológicas de Duelo frente a la pérdida de salud (RPD-PDS-38)

\begin{tabular}{|l|c|c|}
\hline & $\mathrm{N}^{\mathrm{o}}$ & $\%$ \\
\hline Negación & 0 & 0 \\
\hline Ira & 3 & 30 \\
\hline Negociación & 7 & 70 \\
\hline Fe/esperanza & 5 & 50 \\
\hline Depresión & 4 & 40 \\
\hline Aceptación & 1 & 10 \\
\hline Presencia de duelo problemático & 8 & 80 \\
\hline
\end{tabular}

En cuanto a la escala de duelo, los resultados que mostramos en la tabla 4 concuerdan con los hallazgos de Reza et al., (2017) y Velásquez, (2015), con presencia de respuestas de ira en 3 de los casos y depresión en 4 de ellos. Hallamos además respuestas asociadas a la etapa de promesas-negociación en 7 participantes y de fe-esperanza en 5 de ellas. Una persona mostraba respuestas asociadas a la etapa de aceptación. Esta escala no propone puntos de corte en la puntuación total, por lo que no era posible interpretar los datos sin la valoración posterior de la misma paciente. Sí indica que cuando el resultado del afecto negativo es mayor que la actitud positiva, hay indicios de duelo problemático, lo que en la muestra estudiada ocurre en el $80 \%$ de los casos.

Tabla 5. Resultados cuestionario pre-test $(n=10)$

\begin{tabular}{|l|l|}
\hline ¿Tiene dificultad para expresar con palabras cómo & $60 \%$ no tienen dificultades en absoluto. \\
se siente? & $10 \%$ tienen dificultades ocasionalmente. \\
& $33 \%$ casi siempre. \\
\hline ¿Cree que la actividad artística le ayudaría a & $20 \%$ en absoluto. \\
expresar cómo se siente? & $30 \%$ en ocasiones. \\
& $50 \%$ siempre o casi siempre. \\
\hline ¿Le resulta aburrida su rutina hospitalaria? & $10 \%$ en absoluto \\
\hline ¿Sabe qué es el arteterapia y cómo le puede & $90 \%$ siempre o casi siempre \\
ayudar? & $60 \%$ no conocen en que consiste la terapia. \\
\hline ¿Cree que realizar actividades artísticas le & $40 \%$ si tienen algún conocimiento \\
\hline ayudaría a afrontar su nuevo estado de salud? & $40 \%$ en ocasiones \\
\hline ¿Cree que el Arteterapia le puede ayudar de alguna & $60 \%$ casi siempre o siempre. \\
manera? & $20 \%$ en absoluto \\
\hline
\end{tabular}


En los resultados del cuestionario diseñado por el equipo (tabla 5) que pretenden recabar información más específica al respecto del uso del arteterapia, recogimos datos al respecto de las expectativas de recibir la intervención y la utilidad de la misma. Por un lado, 6 personas afirman no tener dificultades en absoluto para expresar con palabras cómo se siente, mientras que 8 participantes creen que la actividad artística le ayudaría a expresar cómo se sienten y específicamente a afrontar su nuevo estado de salud en 6 de los casos.

Si bien no se conoce muy bien en qué consiste la intervención, ni en qué aspectos puede ser beneficiosa, en 8 de los casos, las participantes creen que el Arteterapia puede ayudarles de alguna forma inespecífica.

\subsection{Comparativa pre-post test}

Como se ha señalado anteriormente, 4 pacientes completaron la terapia. A continuación, presentamos el perfil de estas participantes y una comparativa de los datos recogidos en las evaluación previas y posteriores a la terapia.

Tabla 6. Variables sociodemográficas $(n=4)$

\begin{tabular}{|c|c|c|}
\hline Edad media & 65.7 & \\
\hline \multicolumn{3}{|l|}{ Género } \\
\hline Varones & 2 & $50 \%$ \\
\hline Mujeres & 2 & $50 \%$ \\
\hline \multicolumn{3}{|l|}{ Diagnóstico médico } \\
\hline Isquemia crónica & 2 & $50 \%$ \\
\hline Pie diabético & 1 & $25 \%$ \\
\hline Shock séptico & 1 & $25 \%$ \\
\hline \multicolumn{3}{|l|}{ Motivo de ingreso } \\
\hline Amputación mayor & 2 & $50 \%$ \\
\hline Amputación menor & 2 & $50 \%$ \\
\hline
\end{tabular}

Los datos sociodemográficos recogen una edad media de casi 66 años e igual presencia de varones y mujeres. En cuanto al diagnóstico médico, se trata de dos casos de isquemia crónica, uno de diabetes y un shock séptico de origen infeccioso. La magnitud de las amputaciones se reparte en la misma medida entre mayores (aquellas que se producen por encima del tobillo) y menores (producidas por debajo del mismo).

El número de sesiones que recibió cada paciente fue irregular. En el caso de la persona que menos recibió fueron 6 y en el caso del mayor número fueron 20. Las otras dos participantes recibieron 7 y 10 sesiones. 
Tabla 7. Resultados pre-post test de personas que han completado la terapia $(n=4)$

\begin{tabular}{|l|c|l|c|l|}
\hline & \multicolumn{2}{|c|}{ PRE-TEST } & \multicolumn{2}{c|}{ POST-TEST } \\
\hline Beck & 17.5 & Sintom. depres. leve & 13 & Sintom. depres mínima \\
\hline Rosemberg & 27.8 & Autoestima media & 26.5 & Autoestima media \\
\hline Bis & 10.5 & Imagen completa & 4.25 & Imagen completa \\
\hline
\end{tabular}

Según los resultados mostrados en la tabla 7 la sintomatología depresiva pasa de ser leve a mínima. En el caso de la autoestima, aunque se mantiene en los valores considerados como autoestima media, disminuye acercándose a autoestima baja. La escala de imagen corporal indica una mejoría en la misma, situándose al finalizar la intervención más lejos del punto de corte.

Tabla 8. Escala de Respuestas Psicológicas de Duelo

frente a la pérdida de salud (RPD-PDS-38)

\begin{tabular}{|} 
Pre-test \\
\hline Negación & Ira & Negociación & Fe/esperanza & Depresión & Aceptación & Afecto - & Actitud + & Total \\
\hline 12.25 & 12.5 & 27.25 & 16.25 & 10 & 36.5 & 58.25 & 43.25 & 80 \\
\hline Post-test \\
\hline 12 & 10.25 & 18 & 14 & 7 & 32.5 & 56.5 & 32.75 & 89.5 \\
\hline$\geq 17$ & $\geq 15$ & $\leq 17$ & $\leq 10$ & $\geq 10$ & $\leq 25$ & \multicolumn{4}{|c|}{ Af -> Ac +} \\
\hline$\downarrow$ & $\downarrow$ & $\downarrow$ & $\uparrow$ & $\downarrow$ & $\downarrow$ & \multicolumn{1}{|c|}{ Empeora el duelo problemático } \\
\hline
\end{tabular}

Atendiendo a las puntuaciones de la escala de respuestas psicológicas de duelo, podemos observar que disminuyen todas las respuestas psicológicas estudiadas, a excepción de las de fe/esperanza, que aumentan. Aunque disminuye el afecto negativo, también lo hace la actitud positiva, mostrándose la puntuación total más elevada, lo que indica un duelo más problemático.

Tabla 9. Resultados cuestionario pre post-test $(n=4)$

\begin{tabular}{|c|c|}
\hline $\begin{array}{l}\text { ¿Tiene dificultades para } \\
\text { expresar con palabras sus } \\
\text { emociones? }\end{array}$ & $\begin{array}{l}50 \% \text { en absoluto. } \\
50 \% \text { casi siempre. }\end{array}$ \\
\hline $\begin{array}{l}\text { ¿Cree que la actividad } \\
\text { artística le ayudaría a la } \\
\text { expresión emocional? }\end{array}$ & $\begin{array}{l}50 \% \text { en ocasiones. } \\
50 \% \text { siempre o casi } \\
\text { siempre. }\end{array}$ \\
\hline $\begin{array}{l}\text { ¿Le resulta aburrida su } \\
\text { rutina hospitalaria? }\end{array}$ & $\begin{array}{l}50 \% \text { casi siempre } \\
50 \% \text { siempre }\end{array}$ \\
\hline ¿Sabe qué es el arteterapia? & $\begin{array}{l}75 \% \text { en absoluto. } \\
25 \% \text { si tienen algún } \\
\text { conocimiento }\end{array}$ \\
\hline $\begin{array}{l}\text { ¿Realizar actividades artísticas } \\
\text { ayudará a afrontar su nuevo } \\
\text { estado de salud? }\end{array}$ & $\begin{array}{l}50 \% \text { casi siempre } \\
50 \% \text { siempre. }\end{array}$ \\
\hline $\begin{array}{l}\text { ¿Arteterapia le puede } \\
\text { ayudar de alguna manera? }\end{array}$ & $\begin{array}{l}25 \% \text { en absoluto } \\
75 \% \text { siempre. }\end{array}$ \\
\hline $\begin{array}{l}\text { ¿Se ha sentido } \\
\text { acompañado/a por la } \\
\text { Arteterapeuta? }\end{array}$ & $\begin{array}{l}25 \% \text { muchas veces } \\
75 \% \text { siempre }\end{array}$ \\
\hline
\end{tabular}

\begin{tabular}{|l|l|}
\hline $\begin{array}{l}\text { ¿Las sesiones han facilitado } \\
\text { expresar con palabras sus } \\
\text { emociones? }\end{array}$ & $\begin{array}{l}25 \% \text { ocasionalmente } \\
75 \% \text { casi siempre }\end{array}$ \\
\hline $\begin{array}{l}\text { ¿La actividad artística la } \\
\text { ha ayudado a la expresión } \\
\text { emocional? }\end{array}$ & $100 \%$ muchas veces \\
\hline $\begin{array}{l}\text { ¿El arteterapia ha mejorado su } \\
\text { estancia hospitalaria? }\end{array}$ & $\begin{array}{l}25 \% \text { ocasionalmente } \\
75 \% \text { siempre o casi } \\
\text { siempre }\end{array}$ \\
\hline $\begin{array}{l}\text { ¿Sabría explicar qué es el } \\
\text { arteterapia? }\end{array}$ & $\begin{array}{l}75 \% \\
\text { aproximadamente } \\
25 \% \text { totalmente }\end{array}$ \\
\hline $\begin{array}{l}\text { ¿Arteterapia le ha ayudado a } \\
\text { afrontar su nuevo estado de } \\
\text { salud? }\end{array}$ & $\begin{array}{l}25 \% \text { ocasionalmente } \\
75 \% \text { siempre o casi } \\
\text { siempre }\end{array}$ \\
\hline $\begin{array}{l}\text { ¿Arteterapia le ha ayudado de } \\
\text { alguna manera? }\end{array}$ & $\begin{array}{l}25 \% \text { casi siempre } \\
75 \% \text { siempre. }\end{array}$ \\
\hline \multicolumn{2}{|l}{} \\
\hline
\end{tabular}


En los resultados de los cuestionarios diseñados por el equipo que podemos observar en la tabla 9, la mitad de las participantes refería no tener dificultades para expresar con palabras sus emociones, la otra mitad refería tener dificultades casi siempre. Después de la intervención todas las personas atendidas referían que las sesiones habían facilitado la expresión emocional a través de la palabra, en 3 de los 4 casos con una frecuencia casi completa.

A la pregunta de si la actividad artística ayudaría a la expresión emocional, la mitad de la muestra piensa que lo haría en ocasiones y la otra mitad siempre o casi siempre. Una vez realizadas las sesiones, todas las participantes refieren que esta actividad les ha ayudado a expresar sus emociones.

Asimismo, todas las participantes cuando se les pregunta antes de la intervención opinan que la rutina hospitalaria es aburrida siempre o casi siempre. En el cuestionario posterior una de ellas opina que las sesiones han mejorado esta estancia en ocasiones, mientras que las 3 restantes opinan que la ha mejorado siempre o casi siempre.

Antes de comenzar la intervención, 3 de las participantes no tenía conocimiento de en qué consistía la terapia, posteriormente una de ellas refiere conocerlo bien y el resto sólo aproximadamente.

En relación a si la actividad artística ayudaría específicamente a afrontar su nuevo estado de salud todas las participantes tenían la expectativa de que así sería. Al finalizar la intervención, una de ellas opina que lo había hecho ocasionalmente y 3 refieren que siempre o casi siempre.

En cuanto a la expectativa de ayuda inespecífica que podía aportarles el arteterapia, una participante opinaba que en absoluto, mientras 3 referían que siempre. Al finalizar la intervención, todas las participantes expresan que les había aportado una ayuda inespecífica.

Además de todo ello, todas las participantes refieren haberse sentido acompañadas por la arteterapeuta siempre o casi siempre.

\section{Discusión}

Los resultados obtenidos en nuestro estudio arrojan muy variada información al respecto de la efectividad del arteterapia sobre la depresión, la autoestima, la autoimagen y el duelo de las personas amputadas. A través de los cuestionarios diseñados por el equipo, hemos obtenido a su vez información sobre los efectos en otros aspectos que no se contemplaban en las escalas.

En primer lugar, encontramos una gran aceptación para participar en el estudio, lo cual concuerda con los hallazgos de (Jiménez et al., 2017) en cuanto al interés que refieren las personas amputadas por terapias complementarias. Asimismo, según los datos sociodemográficos y clínicos recogidos, en la mayoría de personas atendidas la amputación tiene su origen en una enfermedad previa, lo cual era previsible por el entorno donde realizamos el proyecto y concuerda con una mayor incidencia de estas intervenciones quirúrgicas en las patologías vasculares y diabetes que por causas traumáticas.

En cuanto a los resultados obtenidos en las escalas Beck (depresión), Rosemberg (autoestima) y BIS (imagen corporal), la información recogida en la valoración previa concuerda con los estudios consultados (Atherton, 2002; Ghous, et al., 2015; Tizón, 2004), donde encontramos una prevalencia importante de estas alteraciones 
psicológicas. Si bien la muestra de que disponemos no es significativa, esta concordancia apunta a resultados coherentes. Los datos recogidos en la valoración posterior a la intervención indican una mejoría tanto en la sintomatología depresiva como la percepción de la imagen corporal. En el caso de la autoestima las puntuaciones empeoran. Pensamos que estos resultados pueden apuntar a que las sesiones de arteterapia han facilitado la expresión de emociones habituales en el colectivo como la rabia o el enfado hacia sí mismas por el mal manejo de sus enfermedades previas. Esto puede haber tenido repercusiones complejas en las cuales no se han podido profundizar debido a la corta duración de la intervención.

En cuanto a los resultados obtenidos en la escala de Respuestas Psicológicas de Duelo ante la Pérdida de la Salud (RPD-PDS-38), es necesaria una reflexión sobre el uso de la misma para el presente estudio. Basada en el modelo de Kübler-Ross, (1993) la escala parte de la premisa de que el duelo será más problemático si existen respuestas de negación, ira o depresión, y menos problemático cuando las respuestas presentes son de negociación o esperanza. Este acercamiento es cuestionable desde la visión de la intervención en arteterapia, que pretende facilitar la expresión emocional independientemente del carácter de estas emociones. Así, consideramos que lo interesante de los datos obtenidos con esta escala son los cambios que se producen en los valores de todas las respuestas, lo cual apunta a que las sesiones de arteterapia facilitan el movimiento entre las diferentes etapas.

Por otro lado, los datos obtenidos en los cuestionarios diseñados por el equipo del proyecto, confirman el poco conocimiento de la disciplina en el colectivo así como expectativas positivas de lo que puede ofrecerles. Resulta significativo que esta buena predisposición a los posibles beneficios de recibir la terapia, se ve satisfecho en los resultados de la evaluación posterior, donde todos los datos obtenidos apuntan a una alta satisfacción de la atención recibida. Es destacable la mejora de la estancia hospitalaria, la ayuda en el afrontamiento del nuevo estado de salud y la percepción de ayuda inespecífica. Este último resultado, aunque positivo, sugiere la necesidad de investigaciones que estudien de manera más concreta los beneficios específicos del arteterapia en el colectivo de personas amputadas.

\section{Limitaciones del estudio}

Los resultados de nuestro estudio, si bien apuntan a que la intervención en arteterapia puede ser eficaz en el tratamiento de personas amputadas, requieren a su vez una lectura crítica que pueda ser tenida en cuenta en futuras investigaciones.

En primer lugar, el diseño del proyecto como estudio piloto, no nos ha permitido disponer de una muestra de control que validara nuestros resultados. Durante el desarrollo del mismo, encontramos además algunos imprevistos, que nos llevaron a tomar decisiones que han supuesto limitaciones metodológicas. La estancia hospitalaria breve ha supuesto la pérdida de algunos pacientes, lo cual intentamos minimizar ampliando la intervención no sólo al ámbito del Hospital del Mar sino al Centro Fórum del Parc de Salut Mar al que muchas pacientes son derivadas.

Asimismo, al no poder llegar a realizar el número de sesiones previstas en el diseño del estudio acordamos un máximo de 10 sesiones y un mínimo de 5. Para considerar que se ha completado la terapia se ha de realizar además un cierre tera- 
péutico explicitado como tal con la participante. Esto ha provocado que el número de sesiones sea irregular en cada paciente.

Por otro lado, la muestra de la que disponemos es muy pequeña y por lo tanto no representativa, lo cual es debido a un lado a la falta de recursos humanos que permitieran atender al mismo tiempo a más pacientes y por la pérdida de un gran número de participantes debido a diferentes motivos.

El diseño del proyecto ARPA contempla un estudio cuantitativo. Sin embargo, las limitaciones mencionadas apuntan al interés de realizar un análisis cualitativo de los datos recogidos que pueda arrojar más luz sobre los resultados expuestos ofreciendo una visión más acertada de los efectos del arteterapia en estas personas. Sin embargo, la realización de un análisis sistemático sobre la información recogida en las sesiones supone un nuevo estudio que requiere permisos de los que actualmente no disponemos.

\section{Reflexiones ético-metodológicas}

Es preciso también realizar algunos apuntes sobre cuestiones éticas que puedan ser tenidas en cuenta en futuros estudios.

En primer lugar, no disponemos de un protocolo lo suficientemente claro para la inclusión de participantes en el estudio. Este aspecto ha supuesto dificultades concretas. Un ejemplo claro de ello ha ocurrido en varias ocasiones: cuando la enfermera comunicaba a la persona que recibiría una propuesta; eran comunes preguntas lógicas como ¿y qué me propondrá?, ¿en qué consiste eso? El abordaje de estas estaba inicialmente pensado para ser respondido por la arteterapeuta, lo cual no ha sido siempre así, por lo que algunas pacientes pueden tener la sensación de recibir informaciones confusas, parciales o inexactas sobre qué se les está proponiendo.

Por otro lado, una pregunta que nos han hecho en varias presentaciones es si las personas que no quisieron participar en el estudio pudieron acceder a la intervención. En nuestro caso no planteábamos esta posibilidad y a la vez ninguna paciente nos preguntó sobre ello. Sin embargo, nos parece un aspecto importante a tener en cuenta en el diseño, ya que este posicionamiento puede ser una fuente de exclusión de posibles receptoras de nuestro abordaje. Si ofrecemos intervenciones necesariamente unidas a la participación en investigaciones científicas, debemos ser conscientes de los efectos éticos que esta decisión conlleva.

Por otro lado, la evaluación que diseñamos resulta muy larga y pesada para las participantes, que en prácticamente todos los casos han expresado acabar muy cansadas de responder tantas preguntas. En el futuro, pueden resultar más respetuosas evaluaciones más cortas, bien que evalúen aspectos más concretos o utilicen para ello técnicas más sencillas.

Otra cuestión a la que nos enfrentamos fue al caso de una participante en quien al acercarse el fin de las 10 sesiones previstas, observamos un empeoramiento en el estado emocional general, por lo que decidimos ofrecer la realización de otros 10 encuentros. Si bien somos conscientes de que esto resta rigurosidad a nuestro estudio, nos parece un posicionamiento ético pertinente y necesario, que además sirvió en ese caso para que la participante terminara el proceso con mejores resultados en todas las variables estudiadas. 


\section{Apuntes sobre datos cualitativos}

A continuación, exponemos algunos apuntes sobre la temática del contenido artístico y verbal recogido en las sesiones, destacando que se trata de la visión subjetiva de la arteterapeuta. En ella es importante resaltar que existen diversas particularidades en el proceso de cada paciente, no obstante, es posible observar algunos puntos de encuentro.

De manera general podemos apreciar una necesidad muy acuciante de compartir sus vivencias, los detalles y emociones que suscita la experiencia que han pasado o están pasando. En lo que cuentan son muy habituales las referencias a cuestiones médicas: preocupaciones por la última visita, el pronóstico y la incertidumbre de la evolución del proceso. Aparecen también temas más cotidianos como la opinión sobre la atención de las profesionales del centro e incomodidades de la estancia hospitalaria. En un plano más espiritual aparece la idea de Dios percibido en ocasiones como un apoyo, en ocasiones como juez asociado a sentimientos de responsabilidad e incluso de merecer la situación actual. Este último aspecto está relacionado con sentimientos de culpa por el mal manejo de la propia enfermedad, cuestión que aparece en algunas sesiones de forma simbólica.

En la propuesta artística muchos de los contenidos se relacionan con el proceso de duelo de manera indirecta. Es común la presencia de imágenes referentes a pérdidas sufridas a lo largo de su vida; objetos significativos, el rol profesional o personas ausentes. A partir de estas imágenes ha sido posible llegar a cuestiones más complejas. La distancia que ofrece el trabajo artístico permite abordar emociones como el miedo a la enfermedad y la muerte, una sensación de desamparo en el trato recibido o la culpabilidad con respecto al manejo de su enfermedad. En este sentido, apreciamos la dificultad para enfrentarse al proceso de duelo que se ha puesto en marcha.

Es preciso recordar que la amputación, en varios casos múltiple, ha tenido lugar hace muy poco tiempo, por lo que hay elementos que resultan urgentes para las pacientes, como la compañía de sus seres queridos, los trámites a que deben enfrentarse o el simple descanso y agotamiento que arrastran. Sin embargo, el acompañamiento resulta útil "de alguna manera" como apuntan los resultados del cuestionario que administramos. Estos datos concuerdan con la observación de los procesos individuales, que en todos los casos pudieron elaborar algún aspecto del proceso en el que estaban inmersas.

\section{Conclusiones}

El proyecto Arpa, a pesar de sus limitaciones, supone una aportación pionera en su ámbito de estudio. Si bien se trata de un proyecto humilde consideramos importante su contribución tanto para las personas que hemos atendido como para la disciplina del Arteterapia.

Los datos de participación indican una buena buena acogida del proyecto, reforzada por el interés y agradecimiento de familias e instituciones implicadas. Además, en el año 2016 la revista Rol ofrece su reconocimiento con el premio a la mejor comunicación científica en formato póster en el marco del XXVIII Congreso de Enfermería Vascular y Heridas. En el año 2018 los resultados del 
proyecto han sido presentados en una comunicación oral en la XXX edición del mismo congreso.

Los resultados de las escalas utilizadas son esperanzadores y animan a continuar la investigación. Nuevos estudios de mayor duración permitirían indagar sobre los efectos a medio plazo y/o en otras fases del proceso de duelo. Los datos recogidos por el cuestionario apoyan la evidencia de que las personas amputadas tienen interés en recibir terapias complementarias y que la intervención en Arteterapia les ha resultado satisfactoria.

Pensamos por tanto que nuestra experiencia es relevante y merece ser compartida. Nuestros resultados, análisis y reflexiones pueden ser enriquecedores no sólo para estudios de arteterapia en el terreno de la cirugía vascular, sino también en aquellos llevados a cabo en entornos hospitalarios y con pacientes en proceso de duelo por pérdida de salud.

Son necesarios, sin embargo, nuevos estudios rigurosos que puedan continuar evidenciando el impacto del Arteterapia en esta población dentro de un contexto hospitalario.

\section{Agradecimientos}

El equipo del proyecto deseamos transmitir nuestro más sincero agradecimiento a todas las personas que voluntariamente participaron en este proyecto. Gracias también a sus familias y al personal del Hospital del Mar y centro Fórum por su disposición y amabilidad. Y gracias, muy especialmente a la Fundación de Amigos del Hospital del Mar, por creer en nuestro proyecto y hacerlo posible.

\section{Referencias}

Agea, D., Luis, J., Costa, L., \& Díaz, G. (2013). Suffering of amputees. Etnographic approach with psychotherapeutic applications, 1, 22.

Atherton, R. (2002). Psychological adjustment to lower limb amputation amongst prothesis users.pdf. University of Leicester. Recuperado de https://lra.le.ac.uk/ bitstream/2381/31329/1/U163220.pdf

Case, C., \& Dalley, T. (1992). The handbook of art therapy. London: Routledge.

Collette, N. (2011). Arteterapia y cáncer. Psicooncología, 8(1). https://doi.org/10.5209/rev_ PSIC.2011.v8.n1.7

Collette, N. (2016). Arteterapia en el final de la vida. Arteterapia. Papeles de arteterapia y educación artística para la inclusión social, 10(0). https://doi.org/10.5209/rev_ ARTE.2015.v10.51684

Espinoza, M. J., \& García, D. (2014). Niveles de amputación en extremidades inferiores: repercusión en el futuro del paciente. Revista Médica Clínica Las Condes, 25(2), 276280. https://doi.org/10.1016/S0716-8640(14)70038-0

Flórez, S., León, M., Torres, M., Reyes, F., Serpa, J. C., \& Ríos, A. M. (2009). Manejo farmacológico del dolor neuropático. Revista Colombiana de Anestesiología, 37(4), 356372. https://doi.org/10.1016/S0120-3347(09)74011-0

Ghous, M., Gul, S., Siddiqi, F. A., Pervaiz, S., \& Bano, S. (2015). Prevalence among amputees. Professional Med J, 22(2), 263-266. 
Góngora, V. C., \& Casullo, M. M. (2009). Validación de la escala de autoestima de Rosenberg en población general y en población clínica de la Ciudad de Buenos Aires, 1, 16.

Jiménez, D., Ramírez, A., Rueda, J. C., \& Díaz, J. L. (2017). Analysis of Quality of Life and Pain in Amputee Patients. Revista de Psicología de la Salud (New Age), 5(1), 31.

Juárez, D. M., \& Landero, R. (2014). Imagen corporal, funcionamiento sexual, autoestima y optimismo en mujeres con cáncer de mama. Nova Scientia, 4(7), 17. https://doi. org/10.21640/ns.v4i7.175

Kübler-Ross, E. (1993). Sobre la muerte y los moribundos (4. ${ }^{\text {a }}$ ed.). Barcelona: Ediciones Grijalbo, S.

Luzzato, P., Sereno, V., \& Capps, R. (2003, January). A communication tool for cancer patients with pain: The art therapy technique of the Body Outline. Palliative \& Supportive Care, 1(2), 135-142

Malavera, M. A., Carrillo, S., Gomezese, O. F., García, R. G., \& Silva, F. A. (2014). Fisiopatología y tratamiento del dolor de miembro fantasma. Revista Colombiana de Anestesiología, 42(1), 40-46. https://doi.org/10.1016/j.rca.2013.09.012

Miaja Ávila, M., \& Moral de la Rubia, J. (2014). Desarrollo y validación de la escala de respuestas psicológicas de duelo ante la pérdida de la salud (RPD-PS-38). Psicooncología, 11(2-3). https://doi.org/10.5209/rev_PSIC.2014.v11.n2-3.47395

Orgilles, P. (2015). El chico que se echaba de menos. Arteterapia. Papeles de arteterapia y educación artística para la inclusión social, 9, 99-112. https://doi.org/10.5209/rev_ ARTE.2014.v9.47485

Porta, V. G., \& Retes, R. R. (2013). Manifestaciones del duelo. En El duelo en oncología (pp. 63-83). Sociedad española de oncología médica. Recuperado de https://www.seom.org/ seomcms/images/stories/recursos/sociosyprofs/documentacion/manuales/duelo/duelo08. pdf

Pounsett, H., Parker, K., Hawtin, A., \& Collins, S. (2006, November). Examination of the changes that take place during an art therapy intervention. International Journal of Art Therapy, 11(2), 79-101.

Reza, A. K. G., Rodríguez, M. C. A., \& Zarza, S. S. (2017). Estudio sobre el duelo en personas con amputación de una extremidad superior o inferior. Revista Electrónica de Psicología Iztacala., 20(1), 34-56.

Sanz, J., Perdigón, A. L., \& Vázquez, C. (2003). Adaptación española del Inventario para la Depresión de Beck. CLÍNICA Y SALUD, 34.

Tizón, J. (2004). Pérdida, pena, duelo; vivencias, investigación y asistencia.pdf. Barcelona: Paidós Ibérica.

Tonon da Luz, S., Souza, J., Andrade, M., Ventoza, C., Honório, G., Avila, A., \& Berral, F. (20112). Valoración del síndrome del dolor fantasma en amputados abordaje bio-psicosocial.pdf. Trauma Fund MAPFRE, 23, 176-182.

Velásquez, E. S. (2015). Proceso de duelo tras amputación de miembros inferiores en pacientes adultos. (p. 92). Guatemala: Universidad San Carlos. 